\title{
EVN imaging of the LIRGI sample
}

\section{N. Ramírez-Olivencia*, M. Pérez-Torres, A. Alberdi, R. Herrero-Illana}

Instituto de Astrofísica de Andalucía (IAA-CSIC, Glorieta de la Astronomía s $/ n$, 18008 Granada, Spain)

E-mail: naimrodiaa.es, torreseiaa.es, antxon@iaa.es, herrerodiaa.es

\section{E. Varenius, J. Conway}

Department of Earth and Space Sciences, Chalmers University of Technology, Onsala Space Observatory, 34992 Onsala, Sweden

E-mail: varenius@chalmers.oso.se, john.conway@chalmers.oso.se

The central few hundred parsecs of Ultra/Luminous Infrared Galaxies (U/LIRGs) are hidden from view at most wavelengths due to the presence of large amounts of dust. Very Long Baseline Interferometry (VLBI) observations are a powerful tool to unveil the nature of the sources beyond dust clouds. In fact, radio observations are unaffected by dust, and thanks to the high angular resolution are able to pinpoint the compact components in the central regions of U/LIRGs. Thus, VLBI observations can potentially disentangle the dominant mechanism responsible for the dust heating in U/LIRGs, i.e., an Active Galactic Nucleus (AGN), or a starburst, or a combination of both.

In this contribution we present preliminary results for 12 galaxies from the Luminous Infrared Galaxy Inventory (LIRGI) sample, obtained from observations with the European VLBI Network (EVN) at 6 and $18 \mathrm{~cm}$. We show images from NGC 2623 and Mrk 331. Mrk 331 shows three relatively faint compact components at C-band, consistent with emission from young supernovae and/or supernova remnants. On the contrary, NGC 2623 shows a single, very bright compact component at both wavelengths and a very inverted spectral index, clearly indicating that this is the AGN.

12th European VLBI Network Symposium and Users Meeting,

7-10 October 2014

Cagliari, Italy

\footnotetext{
* Speaker.
} 


\section{Introduction}

(Ultra)Luminous Infrared Galaxies, (U)/LIRGs, have $\mathrm{L}_{\mathrm{IR}[8-1000 \mu \mathrm{m}]} \geq 10^{(12) 11} \mathrm{~L}_{\odot}$. U/LIRGs play a relevant role in Astrophysics due to the following reasons: i) U/LIRGs produce $10 \%$ of the total radiant energy in the local Universe; ii) The majority of the radiated energy by ULIRGs comes from warm dust grains heated by a central power source: an Active Galactic Nucleus (AGN), a starburst, or both; iii) $20 \%$ of the high-mass star-formation in the local Universe is produced by U/LIRGs (see [1]); iv)U/LIRGs are perfect laboratories to study the symbiosis of stars, gas, Black Holes (BH), and variations of the properties of the Interstellar Medium (ISM) within the galaxy. They are also excellent candidates to host stars with a top-heavy Initial Mass Function (IMF); v) Star forming galaxies at high redshift and U/LIRGs seem to be similar, and the higher the redshift the more they contribute to the specific SFR (see [2]).

Since these galaxies are heavily dust-enshrouded, the central source that heats these nuclear regions remains hidden from view at optical wavelengths. Observations at radio wavelengths are able to unveil and resolve these compact sources and, in that way, determine their nature (AGN, $\mathrm{SN}, \mathrm{SNR}, \ldots)$. Medium-size radio arrays are particularly useful to trace the diffuse radio emission in those galaxies (top panel in Fig. 1). However, their poor angular resolution prevents us from resolving the compact radio emission from individual sources. Our group started a long-term effort to observe a significantly large sample of local U/LIRGs, aimed at studying their central regions with milliarcsecond angular resolution, as Arp299. The bottom panel in Fig. 1) shows a zoom-in in the A region of Arp299 observed with the EVN. This region remains unresolved by the VLA observations, whereas the EVN clearly shows a large number of compact objects, most of them young supernovae and/or SNRs.

\section{EVN imaging of the LIRGI sample}

The Luminous Infrared Galaxy Inventory (LIRGI) project (PIs John Conway and Miguel Pérez-Torres) is an eMERLIN (electronic Multi-Element Radio Linked Interferometer Network) Legacy project, aimed at studying the emission from 42 of the brightest U/LIRGs of the Northern hemisphere (see lirgi.iaa.es). This survey is a subset of the IRAS revised Bright Galaxy Observatories that in turn belongs to the NASA Great Observatories (GOALS program). These U/LIRGs are local galaxies $(\mathrm{D} \leq 250 \mathrm{Mpc})$. To ensure the presence of mergers in the sample the lower limit of infrared emission is $\log \left(\mathrm{L}_{\mathrm{IR}} / \mathrm{L}_{\odot}\right)>11.4$. All of them have $\delta \geq 8$ deg to ensure a good u-v coverage by eMERLIN. The eMERLIN LIRGI observations will allow us to trace the diffuse emission of those U/LIRGs, as well as characterize their free-free absorption, measure their magnetic field strengths, obtain dynamical masses and detect spatial variations in both chemical and physical conditions.

However, we need to observe the LIRGI sample with the EVN to unveil through high angular (milliarsecond) resolution the sources responsible for the dust heating in the innermost regions of the ULIRGs' nuclei. We observed a subset of the LIRGI sample with the EVN at two wavelengths, 5.1 (C band) and $1.7 \mathrm{GHz}$ ( $\mathrm{L}$ band), so that we could characterize the nature of the sources through their spectral index $\left(\mathrm{S}_{v} \propto v^{\alpha}\right)$. We observed with the sensitive $1 \mathrm{~Gb} / \mathrm{s}$ recording mode, with an off source $\mathrm{rms} \leq 10 \mu \mathrm{Jy} /$ beam at both bands. A typical observation consisted of 2 hours 


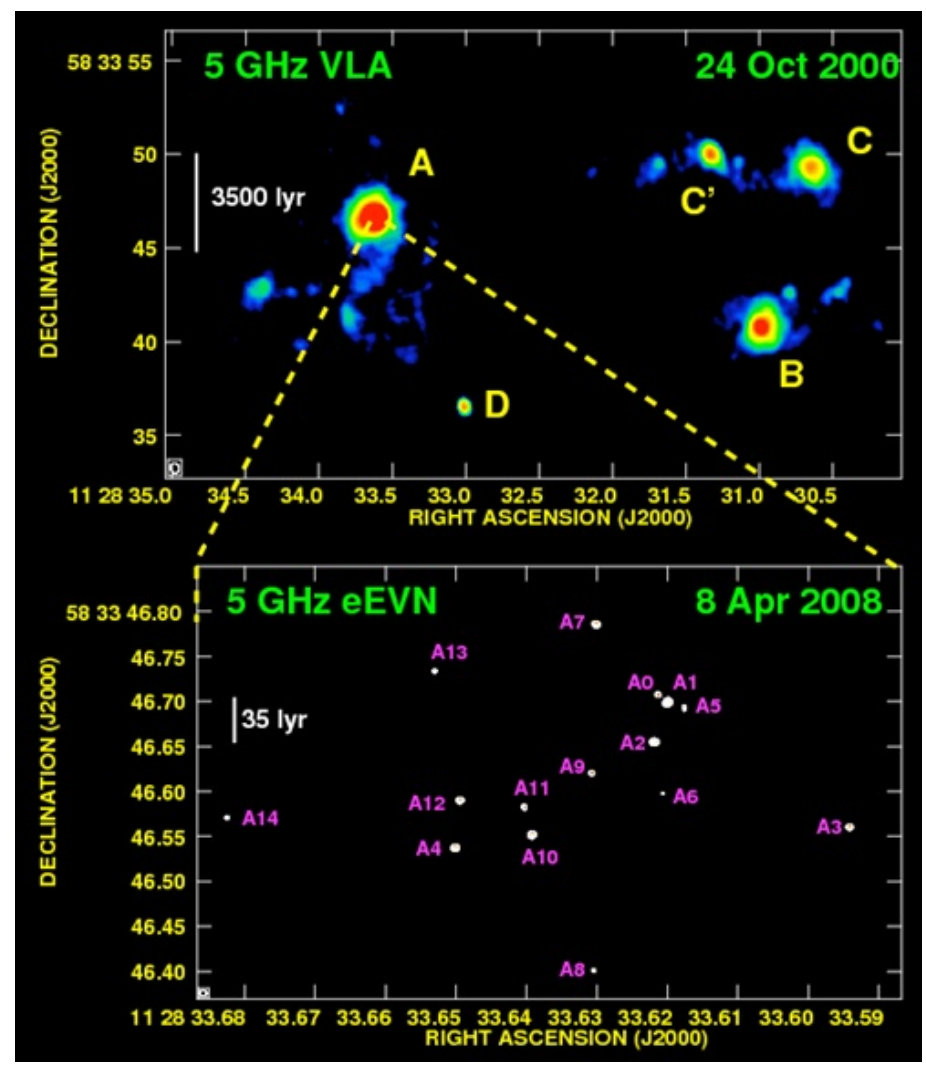

Figure 1: Top:5.1 GHz VLA archival observations of Arp 299, displaying the five brightest knots of radio emission in this merging galaxy. Bottom: Zoom-in of the A-nucleus of Arp299 viewed with the EVN. It unveils many compact sources, among them the AGN (A1) [4] and its respective jet component (A5). Image taken from Pérez-Torres et al. 2009 [3]

per source, with about $80 \mathrm{~min}$ on-target and the remaining $40 \mathrm{~min}$ for phase-refencing, calibration, and antenna slewing. The EVN array typically included the following antennas at both wavelengths: Effelsberg, Westerbork, Jodrell Bank (Lovell), Onsala, Medicina, Noto, Torun, Svetloe, Zelenchukskaya, Badary, Urumqi, and Shanghai. Occasionally, Hartebeesthoek joined the array for the low-declination observations, and in some cases the observations included the Robledo antenna at $\mathrm{L}$ band, and the Yebes antenna at $\mathrm{C}$ band.

\section{Results and Discussion}

We present preliminary results of the EP076A/B (March 2012), EP076C/D (October 2012) and EP088C/D (March 2014) experiments, carried out contemporaneously at C and L band (a total of 12 sources). We considered bona-fide detections those sources with a signal-to-noise ratio above six, except in a few cases where calibration errors were not fully removed. Our preliminary results are summarized in Table 2, and a full account of our EVN-LIRGI observations will be published elsewhere (Ramírez-Olivencia et al, in preparation). We detected 8/12 sources either at C- or Lband (5/12 sources at L-band and 6/12 sources at C-band). Three of the sources (NGC 2623, IRASF10565, and IRAS15250) had detections at both bands. As an example, we show in Figs. 
$2 \mathrm{~b}$ and $2 \mathrm{a}$ the images for Mrk 331 and NGC 2623, obtained from observations on 05/03/2012 and 25/10/2012, respectively.

Mrk 331 (D 70Mpc) is a composite HII/Sy2 galaxy. Our EVN observations detected the source only at $5.1 \mathrm{GHz}$, showing the existence of three clear compact component.The $5.1 \mathrm{GHz}$ monochromatic luminosity of those components (see Table 1) is of the order of a few times $\mathrm{L}_{5 \mathrm{GHz}} \sim 10^{27} \mathrm{erg} \cdot \mathrm{s}^{-1}$. The lower limit to the two-point spectral index is $\alpha \geq 0$ for all of the three sources (assuming a 3- $\sigma$ value for the non-detection at $1.7 \mathrm{GHz}$ ). Those luminosities and (inverted) spectral indices for the sources in the central region of Mrk 331 suggest they may be (relatively) young supernovae, or SNRs, whose low-frequency radio emission is suppressed by the likely existence of $\mathrm{H}$ II regions in the interstellar medium of the host galaxy. In the case Mrk 331 detected sources were SNR, it seems to host a supernova factory in its nucleus, as Arp299 A.

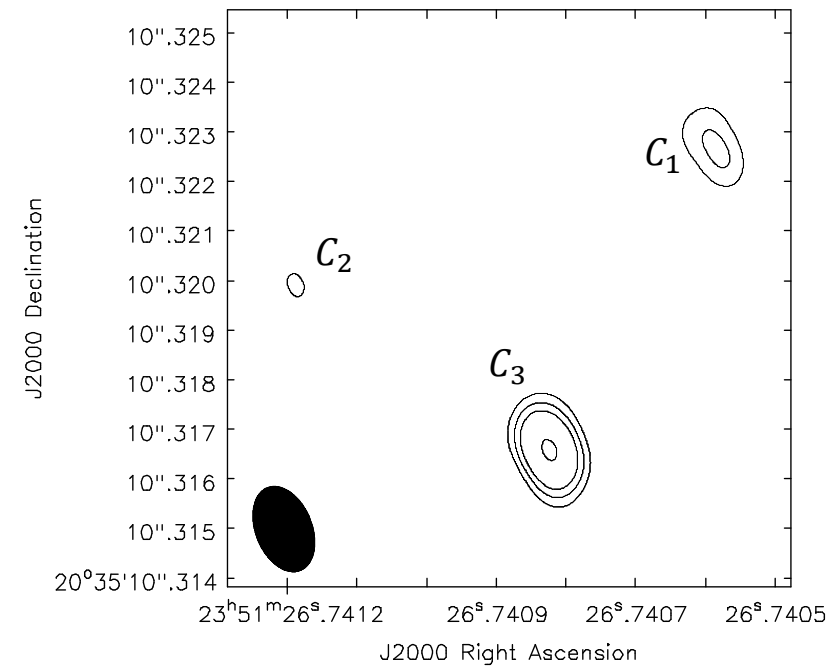

(a) Mrk 331

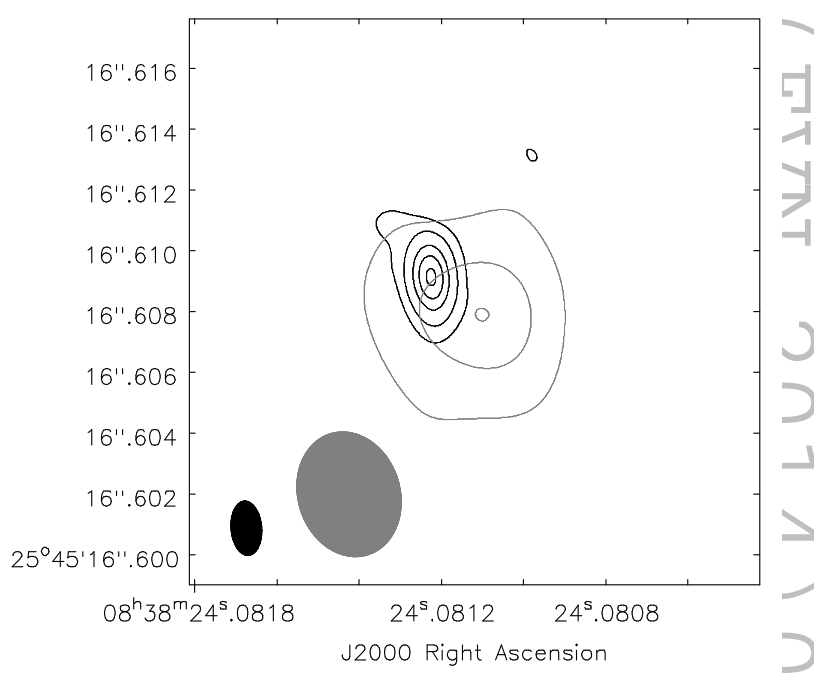

(b) NGC 2623

Figure 2: Left: C-band contours imaging of Mrk 331 LIRG. Contours indicate 5,6,7,10 $\times$ nominal rms. Right: C- (black contours)and L- (gray contours) band imaging of NGC 2623 LIRG. Contours are drawn at 7,20,50,80,110 and 7,20,31 times the nominal rms at C- and L-band, respectively.

NGC 2623 (D 79Mpc) is a composite LINER/Sy2 that hosts a well known AGN (see [5]). Our EVN observations show the existence of a very bright compact component at both wavelengths (see Table 1). Actually, it is the brightest compact source detected so far in our sample. Indeed, the flux density at each wavelength corresponds to a monochromatic luminosity of $\mathrm{L}_{1.7 \mathrm{GHz}}=1.2 \times 10^{29} \mathrm{erg} \cdot \mathrm{s}^{-1} \mathrm{GHz}^{-1}$ and $\mathrm{L}_{5 \mathrm{GHz}}=2.6 \times 10^{29} \mathrm{erg} \cdot \mathrm{s}^{-1} \mathrm{GHz}^{-1}$. The two-point spectral index from our observations is $\alpha=0.67$. Those luminosities are exceedingly high for a recently exploded supernova, or a supernova remnant, and given the inverted spectrum of the source, we conclude that this compact component corresponds to the AGN.

\section{Summary}

We have observed and analysed EVN observations at C- and L-band of the nuclear regions 


\begin{tabular}{cccccc}
\hline \hline $\begin{array}{c}\text { Galaxy } \\
\text { name }\end{array}$ & Object & $\begin{array}{c}\mathrm{rms} \\
{[\mu \mathrm{Jy} / \text { beam }]}\end{array}$ & $\begin{array}{c}\mathrm{S}_{\mathrm{p}} \\
{[\mathrm{mJy} / \text { beam }]}\end{array}$ & $\begin{array}{c}\mathrm{L}_{v} \\
\times 10^{28}\left[\mathrm{erg} \cdot \mathrm{s}^{-1} \mathrm{~Hz}^{-1}\right]\end{array}$ & $\alpha$ \\
\hline Mrk 331 & $\mathrm{C}_{1}$ & 41 & 0.27 & $0.18 \pm 0.03$ & 0.23 \\
& $\mathrm{C}_{2}$ & & 0.22 & $0.14 \pm 0.03$ & 0.04 \\
& $\mathrm{C}_{3}$ & & 0.44 & $0.29 \pm 0.03$ & 0.69 \\
NGC 2623 & $\mathrm{C}$ & 477 & 34 & $26 \pm 7$ & 0.67 \\
& $\mathrm{~L}$ & 286 & 16 & $12 \pm 1$ & \\
\hline
\end{tabular}

Table 1: Summary table for NGC 2623 and Mrk 331 detections. $S_{p}$ is the flux per beam in the peak. For the error of the luminosity we take into account the rms and the $5 \%$ of the luminosity peak. $\alpha$ is defined as $\mathrm{S}_{v} \propto v^{\alpha}$. $\mathrm{C}_{1}, \mathrm{C}_{2}$ and $\mathrm{C}_{3}$ correspond to the detections at $\mathrm{C}$ band of Mrk 331 . In this case, $\alpha$ is calculated with a $3 \sigma$ value for L band ( $\sigma=70 \mu \mathrm{Jy})$. In the case of NGC $2623, \mathrm{~L}$ and $\mathrm{C}$ correspond to the same object and that is why there is only one $\alpha$ value for it.

of 12 galaxies from the LIRGI sample. In most of these sources (8/12) we have detected compact sources at least in one band. Our preliminary results show that most compact sources are relatively weak radio emitters $\left(\mathrm{L} \sim 10^{27} \mathrm{erg} \cdot \mathrm{s}^{-1}\right)$. Some of them show spectral indices that, together with those luminosities, suggest they are likely SNe or SNRs. There are also some cases (e.g., NGC 2623, IRASF10565) where the radio emission is dominated by a single, very bright, compact component $\left(\mathrm{L} \geq 10^{29} \mathrm{erg} \cdot \mathrm{s}^{-1}\right)$, which has a flat, or even inverted spectrum, clearly indicating this is the AGN of the host galaxy.

The EVN-LIRGI project is still ongoing. We are currently analysing additional EVN data corresponding to the remaing 23 sources of the sample (seven sources were already observed by others with high sensitivity VLBI arrays). When all the EVN and eMERLIN data are taken, we will combine those data sets to obtain images of unprecedented detail and sensitivity for the LIRGI sample, ranging from parsec to kiloparsec scales, which will be made available to the whole astronomical community.

\section{Acknowledgments}

The European VLBI Network (EVN) is a joint facility of European, Chinese, South African, and other radio astronomy institutes funded by their national research councils. NRO, MPT, and AA acknowledge support from the Spanish MINECO through grant AYA2012-38491-C02-02. The research leading to these results has received funding from the European Commission Seventh Framework Programme (FP/2007-2013) under grant agreement No 283393 (RadioNet3).

\section{References}

[1] Brinchmann,J., Charlot, S., White, S. D. M., et al. 2004 MNRAS, 351, 1151

[2] Magnelli, B., Elbaz, . D, Chary, et al. 2009 A\& A, 496, 57-75

[3] Pérez-Torres, M. Á., Romero-Cañizales, A., Alberdi, A., Polatidis, A. 2009 A\& A, 507, L17

[4] Pérez-Torres, M. Á., Alberdi, A., Romero-Cañizales, A., Bondi, M. 2010 A\& A, 519, L5

[5] Evans, A. S., Vavilkin, T., Iwasawa, K., et al. 2009 AAS, 21333406E

[6] Weiler, K. W., et al. 1986 ApJ, 301, 790-812 


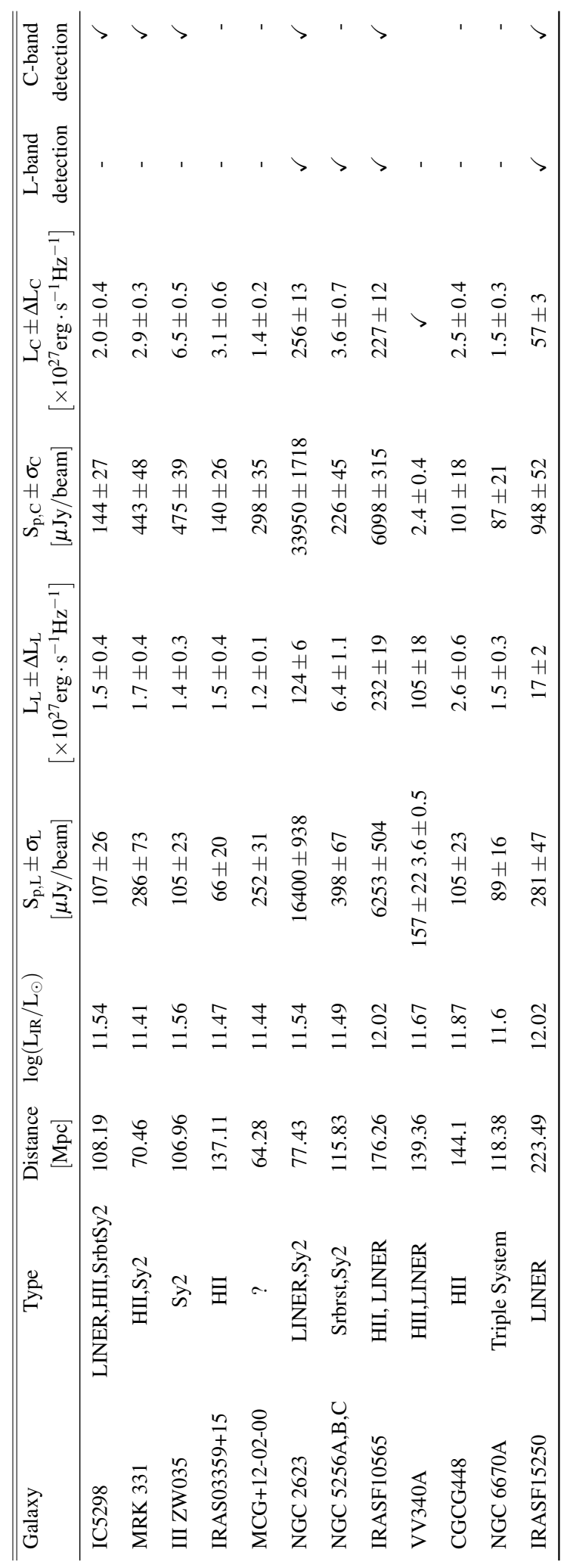

Table 2: Summary table for the 12 U/LIRG sources of the LIRGI sample. The fluxes are measured in the peak of emission, whether or not there is clear detection. For the uncertainty of the flux/luminosity we have take into account the rms and the $5 \%$ of the flux/luminosity peak, at both bands. 